\title{
CONTROL DE GORGOJO (Sitophilus zeamais MOTSCHULSKY) CON POLVOS DE EPAZOTE (Dysphania ambrosioides (L.) MOSYAKIN \& CLEMANTS) EN DIFERENTES GENOTIPOS DE MAÍZ
}

\author{
CONTROL OF WEEVIL (Sitophilus zeamais MOTSCHULSKY) WITH POWDER OF EPAZOTE \\ (Dysphania ambrosioides (L.) MOSYAKIN \& CLEMANTS) IN DIFFERENT GENOTYPES OF MAIZE
}

\author{
Ma. Alma Rangel-Fajardo ${ }^{1}$, J. Ismael Tucuch-Haas ${ }^{1}$, Noel O. Gómez-Montiel ${ }^{2}$, \\ Dianelly de la C. Basto-Barbudo ${ }^{3}$ y Johnny A. Burgos-Díaz ${ }^{1}$
}

\begin{abstract}
'Instituto Nacional de Investigaciones Forestales, Agrícolas y Pecuarias (INIFAP), Campo Experimental Mocochá, Mocochá, Yucatán, México. ${ }^{2}$ INIFAP, Campo Experimental Iguala, Iguala de la Independencia, Guerrero, México. ${ }^{3}$ Instituto Tecnológico de Conkal, Conkal, Yucatán, México.

*Autor de correspondencia (rangel.alma@inifap.gob.mx)
\end{abstract}

\section{RESUMEN}

Durante el manejo postcosecha del maíz (Zea mays L.) se presentan pérdidas importantes por el ataque de plagas de almacén, particularmente por gorgojo (Sitophilus zeamais Motschulsky). El objetivo del presente estudio fue evaluar la efectividad del epazote (Dysphania ambrosioides) para controlar los daños por gorgojo en maíz. Semillas de 11 genotipos de maíz, nueve experimentales y dos híbridos comerciales, todos con humedad de $13 \%$ y sin daño físico que indicara la presencia de insectos, fueron sometidos a tratamientos con polvo de epazote en cuatro concentraciones $\left(0,5,7\right.$ y $10 \mathrm{~g}$ de epazote $\mathrm{kg}^{-1} \mathrm{de}$ semilla) para controlar gorgojo. Se utilizó un diseño completamente al azar con arreglo factorial y tres repeticiones. La unidad experimental consistió en una caja de plástico acondicionada donde se colocaron 100 semillas, previamente pesadas, junto con 50 gorgojos jóvenes y la dosis correspondiente de polvo de epazote. Las variables registradas fueron pérdida de peso, granos dañados y sobrevivencia de insectos, todos expresados en porcentaje. Hubo diferencias entre genotipos de maíz solamente para pérdida de peso $(P \leq 0.05)$; todos los genotipos de maíz registraron pérdidas; sin embargo, el híbrido CLTHW14001 fue el menos afectado con $3.5 \%$ de pérdida de peso. Las dosis de 7 y 10 $\mathrm{g}$ de epazote $\mathrm{kg}^{-1}$ de semilla fueron sobresalientes en las variables pérdida de peso, granos dañados y sobrevivencia de insectos, con $3.4,12.2$ y 3.5 $\%$ en promedio, respectivamente. El híbrido CLTHW14001 mostró el menor daño con la dosis de $7 \mathrm{~g}$ de epazote $\mathrm{kg}^{-1}$ de semilla con $0.94 \%$ de pérdida de peso. El uso de polvo de epazote para el control de gorgojo es efectivo en concentraciones desde $7 \mathrm{~g} \mathrm{~kg}^{-1}$ de semilla. El genotipo de maíz influye en la efectividad del tratamiento.

Palabras clave: Dysphania ambrosioides, Sitophilus zeamais, calidad de grano, plagas de almacén, plantas insecticidas.

\section{SUMMARY}

During postharvest handling of maize (Zea mays L.) significant losses occur due to the attack of storage pests, particularly by weevils (Sitophilus zeamais Motschulsky). The objective of this study was to evaluate the effectiveness of epazote (Dysphania ambrosioides) to control weevil damage in maize. Seeds from 11 genotypes, nine experimental and two commercial, were treated with epazote powder at four concentrations $(0,5,7$ and $10 \mathrm{~g}$ of epazote powder $\mathrm{kg}^{-1}$ of seed) to control weevil. Before treatment, seed moisture content was $13 \%$, and undamaged seeds indicated the absence of insects. A completely randomized design with a factorial arrangement and three replications was used. The experimental unit consisted of a conditioned plastic box where 100 seeds, previously weighed, were placed along with 50 young weevils and the corresponding dose of epazote powder. The variables recorded were weight loss, damaged grains, and insect survival, all expressed as percentages. There were differences between maize genotypes only for weight loss $(P \leq 0.05)$; all the maize populations experienced losses; however, hybrid CLTHW14001 was the least affected with $3.5 \%$ weight loss. Doses of 7 and $10 \mathrm{~g}$ of epazote $\mathrm{kg}^{-1}$ of seed caused reduced weight loss, lower damaged grains, and lower insect survival, at $3.4,12.2$, and $3.5 \%$, respectively. When treated with $7 \mathrm{~g}$ of epazote $\mathrm{kg}^{-1}$ of seed, hybrid CLTHW14001 only lost $0.94 \%$ weight. Epazote powder effectively controls weevil at concentrations from $7 \mathrm{~g} \mathrm{~kg}^{-1}$ of seed. The genotype of maize influences the effectiveness of the treatment.

Index words: Dysphania ambrosioides, Sitophilus zeamais, grain quality, insecticidal plants, storage pests.

\section{INTRODUCCIÓN}

El maíz (Zea mays L.) es un cultivo básico y primordial para la dieta de los mexicanos. El principal destino del cultivo en México es para autoconsumo, primordialmente en regiones de bajos recursos, donde además el maíz tiene importancia religiosa, cultural y económica (SangermánJarquín et al., 2018). Durante el proceso productivo del maíz se presentan pérdidas ocasionadas por plagas, enfermedades o eventos climáticos; sin embargo, se pone poco interés en las pérdidas que se generan durante el almacenamiento (Fernández et al., 2013).

Existen especies de insectos de importancia económica como plagas de semillas y granos almacenados, en México están presentes 25, de los cuales 15 pertenecen a los órdenes Coleóptera y Lepidóptera. Entre los más importantes se encuentran Acanthoscelides obtectus Say (gorgojo del frijol), Sitophilus granarius L. (gorgojo del trigo), Sitophilus zeamais Motschulsky (gorgojo del maíz), Sitotroga cerealella Oliv. (palomilla de los cereales), Ryzopertha dominica Fabricius (taladrillo de los cereales), Prostephanus truncatus (Horn.) (barrenador grande de los granos), Sitophilus oryzae L. (picudo del arroz), Tribolium castaneum Hbst. (gorgojo castaño de la harina), 
Cryptolestes ferrugineus Steph (gorgojo rojizo de los granos), Oryzaephilus surinamensis L. (gorgojo de tórax aserrado), Cryptolestes pusillus Schon. (gorgojo plano de los granos) y Trogoderma granarium Vuelca (gorgojo khapra) (Moreno, 1996).

Los problemas de pérdidas por presencia de plagas de almacén se acentúan aún más en los trópicos; de acuerdo con Bergvinson et al. (2001), las pérdidas de almacén ocasionadas por plagas se asocian con las altas temperaturas y la humedad presente en estas regiones. Suleiman et al. (2015) observaron que granos almacenados a temperaturas mayores de $27^{\circ} \mathrm{C}$ presentaron daños severos de infestación, y las poblaciones del insecto presentaron un crecimiento exponencial. Por otro lado, se han asociado las fechas de siembra con la intensidad de la infestación y daño causado en el grano cosechado; se observó que las fechas tempranas fueron las menos afectadas (Zakka et al., 2015).

Las pérdidas que pueden ocasionar las plagas de almacén se pueden clasificar como pérdidas nutricionales, de peso, fisiológicas y de calidad (Appert, 1987). GarcíaLara y Bergvinson (2007) sugirieron que, para reducir pérdidas, es necesario el desarrollo de variedades resistentes, evaluar la resistencia de las plagas de almacén, mejorar las prácticas tradicionales de conservación y transferir los conocimientos de mejoras en las prácticas de almacenamiento y conservación a los productores.

El uso de extractos naturales o plantas con actividad insecticida es una práctica que se está recuperando de la agricultura de subsistencia (Usha y Devanand, 2011). Entre las plantas que se han empleado para el control insectos están la pimienta (Piper nigrum L.), chile (Capsicum annuum var. longum Sendtn), canela (Cinnamomum zeylanicum Blume), orégano (Origanum vulgare L.), entre otras (Silva et al., 2005; Salvadores et al., 2007). Dichos estudios han tenido el propósito de encontrar productos naturales de bajo costo y fácil acceso para los productores y que además sean amigables con el ambiente (Iannacone et al., 2004).

En maíz se han reportado pérdidas por ataque de plagas, principalmente por Sitophilus zeamais, por arriba del $50 \%$ en materiales susceptibles (Derera et al., 2014). Suleiman et al. (2015) analizaron el comportamiento de esta plaga en siete variedades de maíz, blancos dentados, blancos duros, amarillos duros, palomeros blancos, palomeros amarillos y maíces dulces, y concluyeron que existen materiales del tipo amarillo duro y palomero que presentan potencial para evadir o soportar las pérdidas que genera este insecto. Con base en lo anterior, en el presente estudio se evaluó la eficiencia de control con diferentes concentraciones de polvo de epazote (Dysphania ambrosioides (L.) Mosyakin \& Clemants) sobre Sitophylus zeamais Motschulsky en grano de 11 genotipos de maíz.

\section{MATERIALES Y MÉTODOS}

\section{Material genético}

El estudio se realizó en los laboratorios de fitosanidad del Instituto Nacional de Investigaciones Forestales, Agrícolas y Pecuarias, Campo Experimental Mocochá, Yucatán. Se emplearon semillas de maíz de 11 genotipos, nueve experimentales y dos híbridos comerciales (Cuadro 1). La humedad de las semillas empleadas fue de $13 \%$ en base a peso seco, obtenida antes de establecer el trabajo, con un determinador de humedad portátil John Deere SW08120 (Moline, Illinois, EUA). Las semillas se revisaron para evitar granos dañados, perforados o con galerías que indicaran la presencia de huevecillos o algún estado de desarrollo del insecto.

\section{Polvo vegetal}

Se obtuvieron plantas de epazote (Dysphania ambrosioides (L.) Mosyakin \& Clemants), éstas fueron lavadas con agua potable, se retiró el exceso de agua y se sometieron a secado a $72{ }^{\circ} \mathrm{C}$ en una estufa de secado marca Blue M OV-490a-2 (White Deer, Pennsylvania, EUA), hasta alcanzar peso constante. Las plantas fueron molidas con un molino de mano marca Del Rey y, posteriormente, por un molino eléctrico de marca IKA-Werke M20 (Staufen, Alemania) para obtener polvo fino. Se utilizaron cuatro dosis de polvo de epazote: 0 (testigo), 5, 7 y $10 \mathrm{~g}$ de epazote $\mathrm{kg}^{-1}$ de semilla.

\section{Cría de insectos}

En frascos de plástico con capacidad de $1 \mathrm{~kg}$, con las tapas perforadas y cubiertas por una malla para ventilación, se colocaron semillas de maíz hasta la mitad de su volumen. Los gorgojos fueron colectados en bodegas de almacenamiento de la región, se contaron 1000 ejemplares, los cuales fueron colocados, sin sexar, en los frascos. Se obtuvieron adultos de aproximadamente dos semanas de edad en proporción sexual 1:1 para la infestación de los materiales a evaluar.

\section{Establecimiento del experimento y tratamientos}

En cajas de plástico de $5 \mathrm{~cm}$ de diámetro y $3 \mathrm{~cm}$ de altura, con perforaciones cubiertas de malla de acero, se colocaron 100 semillas, las cuales fueron previamente pesadas en una balanza marca Velab ${ }^{\text {TM }}$ modelo VE-1000 (Ciudad de México), junto con 50 gorgojos en proporción 
Cuadro 1. Descripción de los genotipos de maíz empleados en el estudio.

\begin{tabular}{lccc}
\hline Número & Nombre & Tipo & Color de grano \\
\hline 1 & H-520 & Híbrido & Blanco \\
2 & H-567 & Híbrido & Blanco \\
3 & CLTHW13001 & Híbrido & Blanco \\
4 & CLTHW13002 & Híbrido & Blanco \\
5 & CLTHW13005 & Híbrido & Blanco \\
6 & CLTHW14001 & Híbrido & Blanco \\
7 & CLTHW14003 & Híbrido & Blanco \\
8 & CLTHW14005 & Híbrido & Amarillo \\
9 & CLTHY13002 & Híbrido & Amarillo \\
10 & Sint. Amar. Ocotito & Variedad sintética & Amarillo \\
11 & Sint. Amar. Dentado & Variedad sintética & \\
\hline
\end{tabular}

sexual 1:1. Los tratamientos resultaron de la combinación de los dos factores de estudio, genotipos de maíz (11) y dosis de polvo de epazote (cuatro), lo que dio como resultado 44 tratamientos.

\section{Diseño y unidad experimental}

El diseño del experimento fue completamente al azar con arreglo factorial con dos factores principales y tres repeticiones; el primer factor lo constituyeron los genotipos de maíz (11) y el segundo las dosis de polvo de epazote. La unidad experimental consistió en una caja de plástico acondicionada donde se colocaron 100 semillas, previamente pesadas, junto con 50 gorgojos jóvenes y la dosis correspondiente de polvo de epazote

\section{Variables respuesta}

Los tratamientos se expusieron por un periodo de $15 \mathrm{~d}$ (360 h) y diariamente se monitoreó la sobrevivencia de los insectos, al finalizar el periodo de exposición de 360 horas, se registró la pérdida de peso de las semillas, el número de semillas dañadas o perforadas y el número de insectos vivos. Todas las variables fueron expresadas en porcentaje.

\section{Análisis estadístico}

Los datos fueron transformados con la función arcoseno y se sometieron a análisis de varianza mediante el paquete estadístico SAS versión 9.1 (SAS Institute, 2004). La comparación de medias fue con la prueba de Tukey ( $P$ $\leq$ 0.05) y los resultados se expresaron en las unidades originales.

\section{RESULTADOS Y DISCUSIÓN}

Cuando se realiza el control de plagas de almacén se espera eliminar o reducir las pérdidas, principalmente, en el peso del lote de semillas tratado. En el análisis de los factores principales y sus niveles, los 11 materiales de maíz presentaron reducciones de peso $(P \leq 0.05)$ (Cuadro 2).

El genotipo que registró mayor pérdida de peso fue el Sintético Amarillo Dentado con una reducción de 7.17 puntos porcentuales con respecto a su peso inicial mientras que el material que presentó menor pérdida de peso fue el híbrido CLTHW14001 con 3.52 \%. Para la variable porcentaje de grano dañado no se detectaron diferencias estadísticas, aún cuando las diferencias entre el material más afectado y el de menor daño (Sintético Amarillo Dentado vs. híbrido CLTHW14001) fue mayor a 10 \% (Cuadro 2). La variable sobrevivencia del insecto presentó diferencias $(P \leq 0.05)$ hasta las $264 \mathrm{~h}(11 \mathrm{~d})$; después, todos los genotipos de maíz mostraron similitud estadística $(P \geq 0.05)$ y mantuvieron un intervalo de 25 a 35 $\%$. Los porcentajes más bajos de sobrevivencia del insecto se presentaron en los híbridos H-567 y CLTHW14001, mientras que el Sintético Amarillo Dentado siempre mantuvo un porcentaje alto, entre 78 y $35 \%$, durante el periodo de 24 a $264 \mathrm{~h}$ (Cuadro 3).

Los resultados aquí obtenidos concuerdan con lo observado por Maggioni et al. (2016), quienes reportaron que el crecimiento de las poblaciones de gorgojos y el daño causado fueron diferentes entre variedades; lo que permite identificar que algunas variedades presentan características de tolerancia. Sobre esa base, Lara et al. 
(2018) revelaron que algunas estructuras de la semilla de maíz influyen en el comportamiento e interacción del insecto con el grano, en su estudio detectaron la presencia de componentes químicos en la epicutícula que influyeron en el reconocimiento y atracción como fuente de alimento y reproducción. Por otro lado, Caneppele et al. (2003) concluyeron que existe correlación positiva entre la presencia del gorgojo y la pérdida de peso del lote infestado.

En relación con el factor dosis del polvo de epazote, se registraron diferencias significativas $(P \leq 0.05)$ en el porcentaje de pérdida de peso, en el testigo éste fue de $10.0 \%, 6.9$ puntos porcentuales por arriba del tratamiento de $10 \mathrm{~g}$ de epazote $\mathrm{kg}^{-1}$ de semilla, el cual produjo el menor porcentaje de pérdida de peso (Cuadro 2). Por lo que se refiere a la variable porcentaje de grano dañado, se registraron 17.7 puntos porcentuales de diferencia entre el testigo y el tratamiento de $10 \mathrm{~g}$ de epazote $\mathrm{kg}^{-1} \mathrm{de}$ semilla; sin embargo, esta diferencia fue más drástica en la variable de sobrevivencia del insecto $(P \leq 0.05)$ (Cuadro
3), en la cual el testigo registró $96 \%$ mientras que en el tratamiento de $10 \mathrm{~g}$ de epazote $\mathrm{kg}^{-1}$ de semilla solamente sobrevivió el $2 \%$ de los gorgojos. Las concentraciones de polvo de epazote mantuvieron sus diferencias con respecto al testigo durante todo el periodo de evaluació; no obstante, la concentración de $10 \mathrm{~g}$ de epazote $\mathrm{kg}^{-1}$ de semilla se mantuvo por encima del resto hasta las 240 h $(10$ d); después, las concentraciones de 7 y $10 \mathrm{~g}$ de epazote $\mathrm{kg}^{-1}$ de semilla registraron un intervalo de 1 a 4 $\%$ de sobrevivencia sin diferencias estdísticas $(P>0.05)$ (Cuadro 3).

Con respecto a los resultados de sobrevivencia, Denloye et al. (2010) encontraron que el epazote presentó buen control de gorgojos. Tras trabajar con polvos, extractos y aceite de epazote, dichos autores observaron que el uso de polvo fue el que produjo la menor reducción de peso del lote de granos de maíz tratado al controlar la población de gorgojos y evitar su proliferación; mostraron que esta planta cumple con cuatro propiedades químicas básicas

Cuadro 2. Promedio de las variables pérdida de peso y grano dañado en las semillas de maíz, desglosado por los factores principales de estudio.

\begin{tabular}{|c|c|c|}
\hline Factores principales & Pérdida de peso (\%) & Grano dañado (\%) \\
\hline \multicolumn{3}{|l|}{ Genotipos } \\
\hline $\mathrm{H}-520$ & $4.30 \mathrm{bc}$ & $16.75 a$ \\
\hline $\mathrm{H}-567$ & $5.44 a b c$ & $16.17 \mathrm{a}$ \\
\hline CLTHW13001 & $6.23 a b$ & 18.00 a \\
\hline CLTHW13002 & $4.67 \mathrm{abc}$ & $20.00 \mathrm{a}$ \\
\hline CLTHW13005 & $5.12 a b c$ & 16.33 a \\
\hline CLTHW14001 & $3.52 \mathrm{c}$ & 14.83 a \\
\hline CLTHW14003 & $4.84 a b c$ & 16.33 a \\
\hline CLTHW14005 & $4.76 \mathrm{abc}$ & 16.58 a \\
\hline CLTHY13002 & $6.36 a b$ & 16.58 a \\
\hline Sint. Amar. Ocotito & $6.55 a b$ & $22.42 \mathrm{a}$ \\
\hline Sint. Amar. Dentado & $7.17 \mathrm{a}$ & $24.92 \mathrm{a}$ \\
\hline DSH (0.05) & 2.61 & 10.22 \\
\hline \multicolumn{3}{|l|}{$\begin{array}{l}\text { Dosis } \\
\text { (g de epazote } \mathrm{kg}^{-1} \text { de semilla) }\end{array}$} \\
\hline 0 (testigo) & $10.07 \mathrm{a}$ & $27.88 \mathrm{a}$ \\
\hline 5 & $4.56 b$ & $20.12 b$ \\
\hline 7 & $3.66 \mathrm{bc}$ & $13.55 \mathrm{c}$ \\
\hline 10 & $3.15 c$ & $10.79 \mathrm{c}$ \\
\hline DSH (0.05) & 1.25 & 4.88 \\
\hline
\end{tabular}

Medias con la misma letra en las columnas dentro de cada factor no son estadísticamente diferentes (Tukey, $(\mathrm{P} \leq 0.05)$ ). DSH: diferencia significativa honesta. 


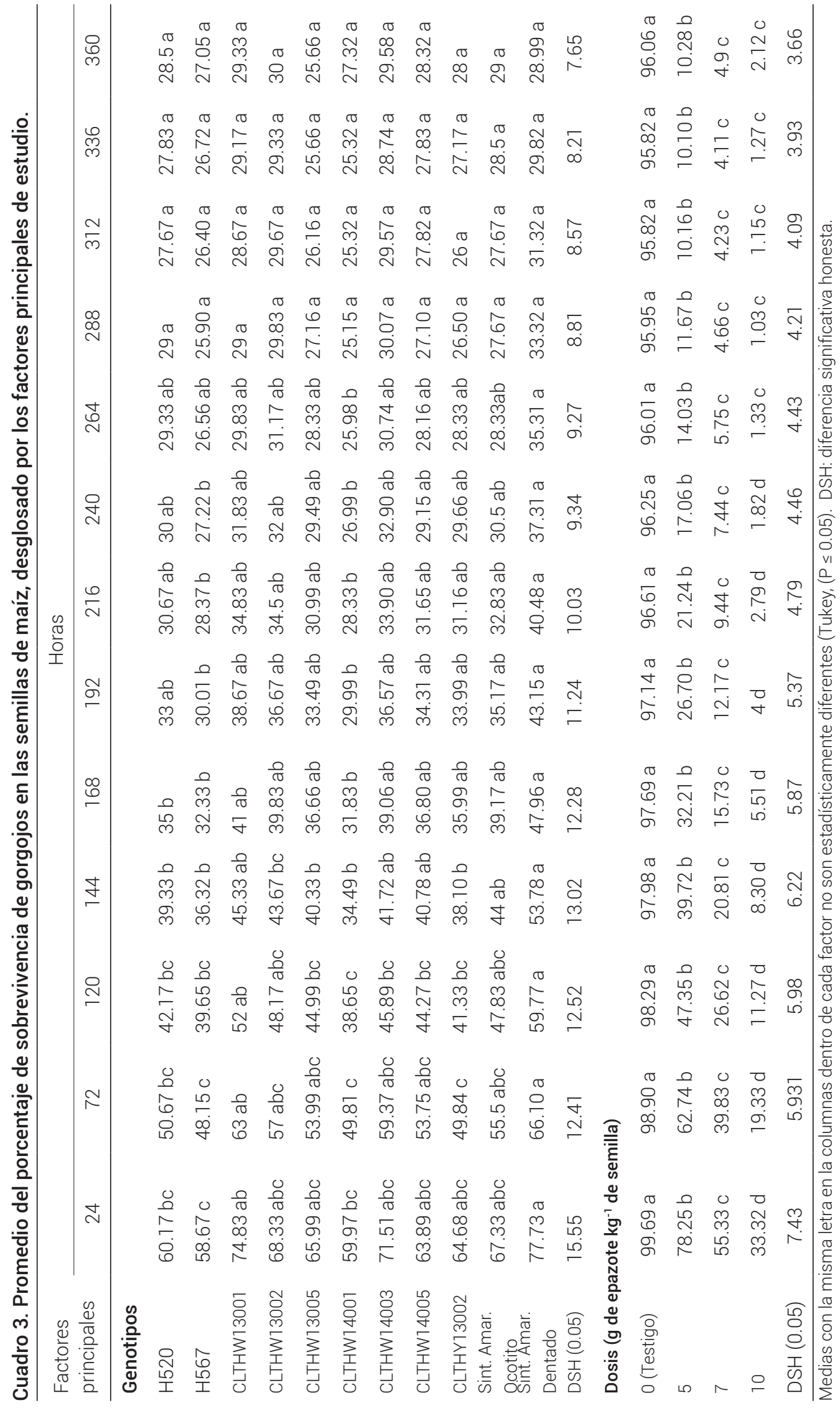




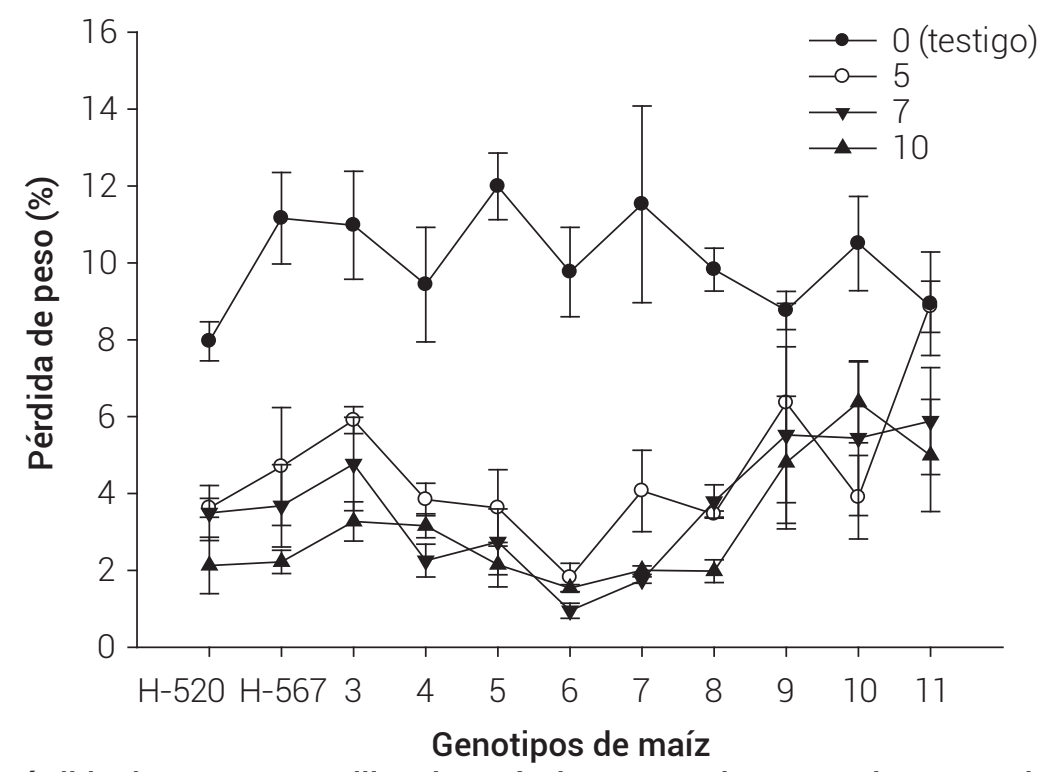

Figura 1. Porcentaje de pérdida de peso en semillas de maíz de 11 genotipos tratados con polvo de epazote a diferentes concentraciones (g de polvo $\mathrm{kg}^{-1}$ de semilla).

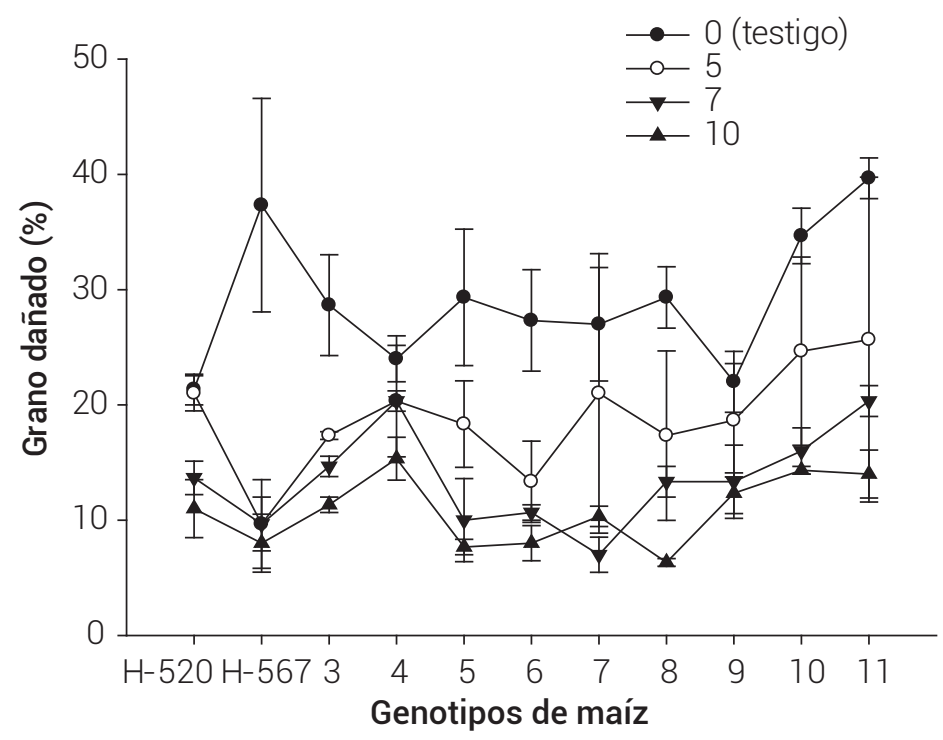

Figura 2. Porcentaje de grano dañado en semillas de maíz de 11 genotipos y tratados con polvo de epazote a diferentes concentraciones ( $\mathrm{g}$ de polvo $\mathrm{kg}^{-1}$ de semilla).

para el control de insectos en granos almacenados: 1) toxicidad para adultos, 2) reducción de oviposición, 3) actividad ovicida y 4) toxicidad en estados inmaduros.

Al observar el efecto de los factores analizados sobre las variables respuesta, todos los genotipos de maíz registraron control favorable del gorgojo, estimado con base en la pérdida de peso (Figura 1). Los materiales con concentración $0 \mathrm{~g}$ de epazote $\mathrm{kg}^{-1}$ de semilla (testigo) tuvieron reducciones de peso de 8 a $12 \%$, lo que concuerda con lo reportado por Caneppele et al. (2003), quienes registraron pérdidas de peso en maíz infestado por gorgojo de $0.36 \%$ por día, en promedio, mientras que Derera et al. (2014) observaron pérdidas de peso de 19 a 56 \% después de cinco meses de infestación.

Los materiales de los tratamientos de 5, 7 y $10 \mathrm{~g}$ de epazote $\mathrm{kg}^{-1}$ de semilla mantuvieron reducciones de peso por debajo del testigo, y aquellos con menor pérdida de peso fueron $\mathrm{H}-520, \mathrm{H}-567$ y CLTHW14001. Con base en lo anterior, Silva et al. (2005) reportaron pérdida de peso de $6 \%$ en semillas de maíz tratadas con epazote y una 
a)

b)
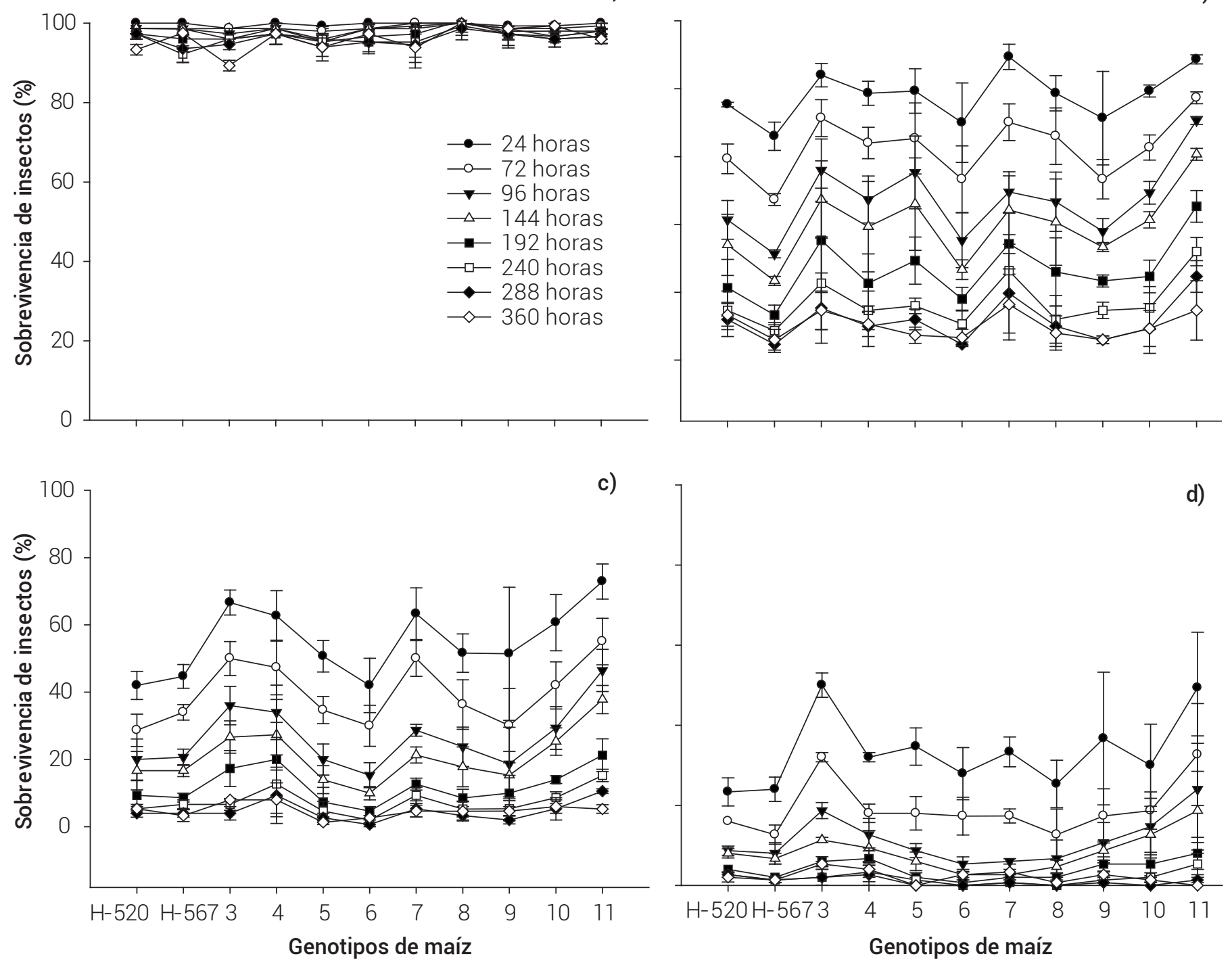

Figura 3. Sobrevivencia de gorgojos tratados con polvos de epazote en diferentes tiempos a través del periodo de

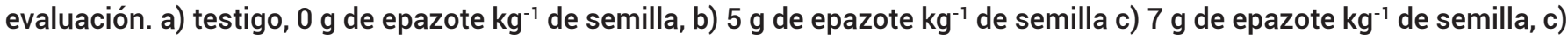

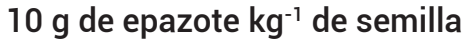

eficiencia en la mortalidad del $65 \%$, mientras que Silva et al. (2003) registraron $0.09 \%$; estos resultados concuerdan con lo obtenido en esta evaluación, donde el material menos afectado registró 0.9 \% de reducción con una concentración de $7 \mathrm{~g}$ de epazote $\mathrm{kg}^{-1}$ de semilla.

Los genotipos de maíz con mayor pérdida de peso, a pesar del tratamiento, fueron el híbrido CLTHY13002 y los sintéticos Amarillo Ocotito y Amarillo Dentado; solamente el Sintético Amarillo Dentado con el tratamiento de $5 \mathrm{~g}$ de epazote $\mathrm{kg}^{-1}$ de semilla registró el mismo comportamiento que su homólogo en el testigo. En cuanto al porcentaje de grano dañado, los genotipos de maíz respondieron de manera diferente en función de la concentración de polvo empleado (Figura 2). Todos los materiales registraron reducciones en el porcentaje de daño; el H-567 sobresalió, al reducir entre 28 y 29 puntos porcentuales el daño en todos los tratamientos con epazote.

La combinación de factores para la variable de sobrevivencia de gorgojos registró para el nivel de $0 \mathrm{~g}$ de polvo de epazote $\mathrm{kg}^{-1}$ de semilla, entre 90 y $100 \%$ de insectos vivos durante las 360 h que duró la evaluación (Figura 3a), mientras que con la concentración de 5 $\mathrm{g}$ de epazote $\mathrm{kg}^{-1}$ de semilla se obtuvo entre 6 y $12 \%$ de sobrevivencia, dependiendo del material (Figura 3b). El nivel de $7 \mathrm{~g}$ de epazote $\mathrm{kg}^{-1}$ de semilla registró reducciones pronunciadas de sobrevivencia desde las 24 
h, la diferencia del material con menor afectación fue de 67 puntos porcentuales de las 24 a las 360 h (Figura 3c); en tanto que la reducción del nivel de $10 \mathrm{~g}$ de epazote $\mathrm{kg}^{-1}$ de semilla fue efectiva desde las $24 \mathrm{~h}$, ya que el porcentaje de sobrevivencia mayor fue de $49 \%$ para el Sintético Amarillo Dentado y 360 h después para ese mismo material no había sobrevivientes (Figura $3 d$ ). Con estos resultados es clara la evidencia del efecto insecticida del epazote sobre el gorgojo; sin embargo, la efectividad se ve afectada por los atributos genéticos del material que se esté tratando. Algunos autores indican que el poder de control con el uso de polvos está influenciado por el tamaño de la partícula del polvo, que puede actuar mediante la obstrucción de los espiráculos de los insectos, los cuales mueren por asfixia (Denloye et al., 2010). Aunque en el presente estudio no se tuvo evidencia de ello, Ofuya y Dawodu (2002) demostraron que existe una relación directa entre el tamaño de las partículas del polvo de plantas empleados y la mortalidad de los insectos; sin embargo, Denloye et al. (2010) reportaron que existe acción ovicida y suprime la oviposición, lo que indica una excelente oportunidad para la protección de granos almacenados.

Cabe mencionar que existe evidencia del efecto de sustancias químicas emitidas por el grano que desencadenan una interacción entre el insecto y el grano (Lara et al., 2018), lo que puede influir directamente en la intensidad de la infestación. Nwosu (2016) sugirió que las variedades con altos niveles de fibra cruda, ácidos fenólicos e inhibidores de tripsina generan condiciones de antixenosis y antibiosis, mientras que los materiales con altos niveles de proteína, almidón y minerales son susceptibles. Existen otras características, como el color o el tamaño, que también influyen en la preferencia del insecto sobre el grano (De Groote et al., 2017).

\section{CONCLUSIONES}

El epazote (Dysphania ambrosioides (L.) Mosyakin \& Clemants) presenta actividad insecticida eficiente para el control de gorgojo en granos de maíz. Las concentraciones de polvo de 7 y $10 \mathrm{~g}_{\text {de epazote }} \mathrm{kg}^{-1}$ de semilla son efectivas para el control de gorgojo. La eficiencia en el control se ve afectada por el genotipo de maíz; sin embargo, desde las 24 horas de aplicación es evidente el efecto del epazote sobre la sobrevivencia de los insectos en todos los genotipos evaluados.

\section{AGRADECIMIENTOS}

Al Instituto Nacional de Investigaciones Forestales, Agrícolas y Pecuarias (INIFAP) por el apoyo con financiamiento fiscal otorgado al proyecto "Respuesta agronómica y postcosecha de variedades e híbridos de maíz, generados por INIFAP, para su impulso en la Península de Yucatán." SIGI 11432834007.

\section{BIBLIOGRAFÍA}

Appert J. (1987) The Storage of Food Grains and Seeds. MacMillan. London, England.146 p.

Bergvinson D. J., S. García-Lara, A. Ramputh, A. Burt and J. T. Arnason (2001) Biochemical and genetic basis for storage pest resistance in maize. In: XI Curso Internacional de Actualización en Tecnología de Semillas, Postcosecha de Granos y Semillas. M. E. Vázquez B., F. Facio P., A. Valdez O. y J. A. Daniel G. (eds.). Saltillo, Coahuila, México. pp:58-66.

Caneppele M. A. B., C. Caneppele, F. A. Lázzari and S. M. N. Lázzari (2003) Correlation between the infestation of level Sitophilus zeamais Motschulsky, 1855 (Coleoptera, Curculionidae) and the quality factors of stored corn, Zea mays L. (Poaceae). Revista Brasileira de Entomologia 47:625-630, https://doi.org/10.1590/S0085-56262003000400015

De Groote H., B. De Groote, A. Y. Bruce, C. Marangu and T. Tefera (2017) Maize storage insects (Sitophilus zeamais and Prostephanus truncatus) prefer to feed on smaller maize grains and grains with color, especially green. Journal of Stored Products Research 71:72-80, https://doi.org/10.1016/j.jspr.2017.01.005

Denloye A. A., W. A. Makanjuola, 0. K. Teslim, O. A. Alafia, A. A. Kasali and A. O. Eshilokun (2010) Toxicity of Chenopodium ambrosoides L. (Chenopodiaceae) products from Nigeria against three storage insects. Journal of Plant Protection Research 50:379-384, https://doi.org/10.2478/v10045-010-0064-7

Derera J., K. V. Pixley, D. P. Giga and I. Makanda (2014) Resistance of maize to the maize weevil: III. Grain weight loss assessment and implications for breeding. Journal of Stored Products Research 59:24-35, https://doi.org/10.1016/j.jspr.2014.04.004

Fernández S. R., L. A. Morales C. y A. Gálvez M. (2013) Importancia de los maíces nativos de México en la dieta nacional. Una revisión indispensable. Revista Fitotecnia Mexicana 36:275-283 https://doi.org/10.35196/rfm.2013.3-S3-A.275

García-Lara S. y J. D. Bergvinson (2007) Programa integral para reducir pérdidas de poscosecha en maíz. Agricultura Técnica en México 33:181-189.

Iannacone J., H. Ayala, J. Álvarez, O. Leyva y E. Bajalque (2004) Cuatro plantas biocidas sobre Sitophilus zeamais y Stegobium paniceum en el Perú. Wiñay Yachay 8:16-27.

Lara U. V., J. S. Dambolena, C. Merlo, M. L. Peschiutta and M. P. Zunino (2018) Insect-corn kernel interaction: chemical signaling of the grain and host recognition by Sitophilus zeamais. Journal of Stored Products Research 79:66-72, https://doi.org/10.1016/j.jspr.2018.08.002

Maggioni K., L. Barboza S., Z. Fernandes X., C. de Bortoli M., L. R. Barros D. and B. E. Pavan (2016) Performance of populations of Sitophilus zeamais Motschulsky (Coleoptera: Curculionidae) on different varieties of maize. African Journal of Agricultural Research 11:873-881, https://doi.org/10.5897/AJAR2015.10505

Moreno M. E. (1996) Análisis Físico y Biológico de Semillas Agrícolas. Tercera edición. Universidad Nacional Autónoma de México. México, D.F. 393 p.

Nwosu L. C. (2016) Chemical bases for maize grain resistance to infestation and damage by the maize weevil, Sitophilus zeamais Motschulsky. Journal of Stored Products Research 69:41-50, https://doi.org/10.1016/j.jspr.2016.06.001

Ofuya T. I. and E. O. Dawodu (2002) Aspects of insecticidal action of Piper guineense Schum and Thonn fruit powders against Callosobruchus maculatus (F.) (Coleoptera: Bruchidae). Nigerian Journal of Entomology 19:40-50.

Salvadores U. Y., G. Silva A., M. Tapia V. y R. Heep G. (2007) Polvos de especias aromáticas para el control del gorgojo del maíz, Sitophilus zeamais Motschulsky, en trigo almacenado. Agricultura Técnica (Chile) 67:147-154, https://doi.org/10.4067/S0365-28072007000200004

Sangermán-Jarquín D. M., M. De la 0-Olán, A. J. Gámez-Vázquez, A. NavarroBravo, M. Á. Ávila-Perches y R. Schwentesius-Rindermann (2018) Etnografía y prevalencia de maíces nativos en San Juan Ixtenco, Tlaxcala, con énfasis en maíz ajo (Zea mays var. 
tunicata a. St. Hil.). Revista Fitotecnia Mexicana 41:451-459, https://doi.org/10.35196/rfm.2018.4.451-459

SAS Institute (2004) SAS/STAT ${ }^{\oplus} 9.1$ User's Guide. SAS Institute Inc., Cary, North Carolina, USA. 5121 p.

Silva G., A. Lagunes y J. Rodríguez S. (2003) Control de Sitophilus zeamais (Coleóptera: Curculionidae) con polvos vegetales solos y en mezcla con carbonato de calcio en maíz almacenado. Ciencia e Investigación Agraria 30:153-160.

Silva G., O. Orrego, R. Hepp y M. Tapia (2005) Búsqueda de plantas con propiedades insecticidas para el control de Sitophilus zeamais en maíz almacenado. Pesquisa Agropecuária Brasileira 40:11 17, https://doi.org/10.1590/S0100-204X2005000100002

Suleiman R., K. A. Rosentrater and C. J. Bern (2015) Evaluation of maize weevils Sitophilus zeamais Motschulsky infestation on seven varieties of maize. Journal of Stored Products Research 64:97102, https://doi.org/10.1016/j.jspr.2015.09.005

Usha R. P. and P. Devanand (2011) Efficiency of different plant foliar extracts on grain protection and seed germination in maize. Research Journal of Seed Science 4:1-14 https://doi.org/10.3923/riss.2011.1.14

Zakka U., N. E. S. Lale and O. C. Umeozor (2015) Efficacy of combining varietal resistance with harvest time and planting date for the management of Sitophilus zeamais Motschulsky infestation in stored maize. Journal of Stored Products Research 60:31-35, https://doi.org/10.1016/j.jspr.2014.10.005 
